Informe Breve

\title{
Desarrollo de una Escala de Interés en Animales
}

\section{Development of a Scale for Interest in Animals}

\section{Gabriel Cirino Gerena ${ }^{1 *}$, \& Kelvin Mariani-Escalante ${ }^{2}$}

1 Test Innovations, San Juan, Puerto Rico. (D) https://orcid.org/0000-0002-3456-7107

2 Test Innovations, San Juan, Puerto Rico. (D) https://orcid.org/0000-0002-8884-0092

* Correspondencia: gcirino@testinnovations.com

Recibido: 7 mayo 2020 | Aceptado: 8 junio 2020 | Publicado: 15 julio 2020

WWW.REVISTACARIBENADEPSICOLOGIA.COM

\section{Citar como:}

Cirino, G., \& Mariani-Escalante, K. (2020). Desarrollo de una escala de interés en animales. Revista Caribeña de Psicología, 4(2), 114-119. https://doi.org/10.37226/rcp.v4i2.3739

\section{RESUMEN}

A pesar de que hay un incremento de actividades terapéuticas que involucran animales y del uso de estos en actividades recreativas y de empleo, los psicólogos, particularmente en Puerto Rico, no han realizado suficientes investigaciones sobre las relaciones de los humanos y los animales. Por otro lado, los inventarios de intereses principales generalmente no incluyen escalas relacionadas con animales. Debido a esta situación decidimos desarrollar una escala de interés en animales para incluirla en el Inventario Cirino de Intereses (ICI). Redactamos una escala experimental con una sub-escala de orientación afectiva (6 ítems) y otra de orientación utilitaria (6 ítems) hacia los animales. El ICI se administró en línea a 1,858 participantes, de los cuales el 48.2\% $(n=895)$ son mujeres y el 51.8\% $(n=963)$ hombres. Ambas sub-escalas resultaron confiables. Las sub-escalas correlacionaron $r=.79$ entre sí, por lo que entendimos que no hay evidencia de dos orientaciones distintas hacia los animales. Correlacionamos la escala final de interés en animales (10 ítems) con las escalas de Interés en Personas $(r=.43)$ e Interés en Objetos $(r=.44)$ del ICI-PR. Finalmente, incluimos la escala de interés en animales como parte de las escalas básicas de Interés en Objetos.

Palabras Claves: desarrollo de escalas; inventario de intereses; interés en animales; relación humano-animal

\begin{abstract}
Psychologists, particularly in Puerto Rico, have not done enough research on the relationships between humans and animals. Although there has been an increase in therapeutic activities involving animals and their use in recreational and employment activities, on the other hand, major interest inventories generally do not include scales related to animals. Due to this situation, we decided to develop a scale of interest in animals to be included in the Cirino Interests Inventory (ICI). We created an experimental scale with a sub-scale of affective orientation (6 items) and another sub-scale of utilitarian orientation (6 items) towards animals. The ICI was administered online to 1,858 participants, of which $48.2 \%(n=895)$ are women and $51.8 \%(n=963)$ men. The correlation of the sub-scales was $\mathrm{r}$ $=0.789$, so we understood that there is no evidence of two different orientations towards animals. We correlate the final scale of interest in animals (10 items) with the general scales of Interest in People $(r=.43)$ and Interest in Objects $(r=.44)$ of the ICI. Finally, we include the scale of interest in animals as part of the basic scales of Interest in Objects.
\end{abstract}

Keywords: human-animal relationship; interest inventory; interest in animals; scale development 


\section{INTRODUCCIÓN}

La relación de los seres humanos con los animales ha sido siempre ambigua. Desde la prehistoria el ser humano incluyó a los animales como fuente de alimentación y compañía. También utilizaron los animales para ayudar en las labores del campo, transportación y los criaron para usarlos en su alimentación (Gutiérrez et al., 2007). Además de lo anterior, existen múltiples actividades donde los animales se utilizan con fines educativos, como son los zoológicos, y actividades de recreación como son los deportes ecuestres y las carreras. Por otro lado, hay deportes donde se da muerte a los animales como son la cacería, las peleas de gallos, las corridas de toros y otras similares. Finalmente, los animales se utilizan en la investigación científica que incluye la observación de la conducta y experimentos donde se viola la integridad física de los mismos. Como resultado de toda esta experiencia de utilizar animales para el consumo, el trabajo y los deportes, ha surgido en muchas personas una orientación utilitaria de los animales y muchas personas se interesan en los animales como objetos.

Sin embargo, la domesticación de animales ha resultado en una oportunidad de convivencia que permite compartir experiencias y realizar observaciones más prolongadas de animales individuales, particularmente con las mascotas. Esta convivencia con mascotas y animales de apoyo, resulta en una experiencia cualitativamente distinta a la anterior. Muchos reconocen que los animales, particularmente las mascotas y animales de apoyo tienen algunas características psicológicas similares a los humanos. Se reconoce que poseen cierto grado de inteligencia, sentimientos y emociones, que sufren física y emocionalmente, y que parecen albergar algunos valores. Algunos parecen tener necesidades psicológicas similares a las de los seres humanos, como son las de afiliación, autonomía y de competencia. Además, hay evidencia de que la relación con ciertos animales puede ser terapéutica para los seres humanos quienes tienden a desarrollar vínculos afectivos muy fuertes con sus mascotas o animales de apoyo (Younggren et al., 2016).

Estas personas llegan a tener cierto grado de identificación con sus animales y desarrollan vínculos de afecto y lealtad hacia ellos. Los niños y niñas pequeños/as parecen tener una afinidad particular con los animales y prefieren jugar con ellos a jugar con juguetes (LoBue et al., 2013). Esta afinidad es más evidente con los animales que se utilizan como mascotas o para fines terapéuticos (Cabán et al., 2014). También los animales contribuyen a reducir la soledad, proveen apoyo emocional como acompañantes (Maharaj, 2016), y logran establecer vínculos de afecto recíproco con niños y niñas autistas (Carlisle, 2014). Estas experiencias han contribuido al surgimiento de una orientación humana de los animales y el interés de muchas personas en los animales es similar al interés en las personas.

Existen varias profesiones y ocupaciones que se relacionan con la salud, atención, manejo y crianza de animales y el número de personas cuyo trabajo se relaciona con animales va en aumento (Gutiérrez et al., 2007). El criterio más importante en la selección de una carrera son los intereses (Cirino Gerena, 2013), sin embargo, hemos encontrado muy poca investigación sobre la naturaleza del interés en animales. De acuerdo a Meléndez Samó (2014) los psicólogos puertorriqueños han evidenciado poco interés en examinar este vínculo humano-animal. Una situación similar prevalece en los Estados Unidos ya que a pesar que se administran millones de inventarios de intereses cada año (Hansen, 1990, 1995), solamente uno de los principales inventarios de intereses publicados, el Campbell Interest and Skill Survey, incluye una escala relacionada: animal care (Campbell, 2002).

Dos de los tres principales inventarios de intereses, el Strong Interest Inventory (Harmon et al., 1994) y el Campbell Interest and Skills Survey (Campbell et al., 1992) adoptan una estructura jerárquica que incluye varias escalas amplias y bajo estas, un grupo de escalas básicas. El tercer inventario principal, el de Holland (1997) solamente cuenta con seis escalas amplias. Igualmente, en Puerto Rico el principal inventario de intereses desarrollado y normalizado aquí (ICI-AE) adoptó recientemente una estructura teórica de 5 intereses amplios o primarios para agrupar sus intereses básicos (Cirino \& Ortíz, 2019) que se presume es universal. Esta consiste de las siguientes orientaciones primarias: orientación hacia personas, objetos, datos, ideas artísticas e ideas realistas en las que se organizan los 13 intereses básicos que tradicionalmente ha tenido dicho inventario. 
Cirino Gerena (2012) propuso que las orientaciones primarias surgen en la infancia y con el tiempo se ramifican en una serie de intereses básicos, los cuales son más específicos que los primarios. La orientación hacia objetos incluye actividades relacionadas con el estudio, manipulación, modificación y reparación de objetos inertes (por ejemplo, piedras, ríos, hierro) y cosas vivientes (por ejemplo, plantas, árboles). Esta orientación hacia objetos parece tener relación con la orientación utilitaria hacia los animales. La orientación primaria hacia personas incluye actividades que están dirigidas a proveer ayuda, educación, orientación y sostén emocional a los demás, por lo que parece tener relación con la hipotética orientación afectiva hacia los animales.

Es evidente la utilidad que tendría una escala para medir interés en animales, por lo que nos preguntamos si la ausencia de dicha escala en la mayoría de los inventarios responde a una omisión o a que no es posible desarrollar una escala homogénea debido a la presunta diferencia de orientaciones hacia los animales. Por tal razón, establecimos dos objetivos para esta investigación: 1) examinar si la relación humano-animal es inherentemente ambigua por lo que es necesario desarrollar dos escalas de intereses, y 2) desarrollar una o dos escalas de interés en animales que sea confiable y obtener alguna evidencia de su validez.

\section{Objetivos de Investigación}

Para examinar la ambigüedad del interés en animales desarrollamos dos sub-escalas experimentales de interés en animales para medir la orientación utilitaria y afectiva. Los objetivos específicos fueron los siguientes: (1) ambas sub-escalas deben ser confiables (con un coeficiente alfa no menor de .70); y (2) las subescalas deben ser relativamente independientes una de la otra, o sea que correlacionarán moderadamente entre sí (correlación entre .50 a .65).

Partiendo de los resultados de la primera parte, el objetivo general fue obtener alguna evidencia de la validez de constructo de las sub-escalas o de la escala total que se obtenga. Los objetivos específicos fueron los siguientes: (1) correlacionar la escala o escalas obtenidas con las escalas de Orientación hacia Objetos y Orientación hacia Personas del ICI-PR; y (2) dicha correlación será moderada (correlación entre .50 a .65).

\section{MÉTODO}

\section{Diseño de Investigación}

Para alcanzar los objetivos de investigación, utilizamos un diseño de estudio instrumental (Ato et al., 2013).

\section{Participantes}

La muestra consistió de 1, 858 participantes de los cuales el $48 \%$ (894) se identificaron como femeninos y el 52\% (962) como masculinos. El promedio de edad de la muestra fue 14.7 (3.4) y el promedio de grado que cursa la muestra es 9.8 (2.5). La distribución por edades y por grados se puede ver en la Tabla 1.

\section{Tabla 1}

Datos básicos de los participantes.

\begin{tabular}{cc|cc}
\hline Edad & $\mathrm{n}(\%)$ & Escolaridad & $\mathrm{n}(\%)$ \\
\hline Féminas & & & \\
12 & $132(14.7 \%)$ & 8vo grado & $307(34.3 \%)$ \\
$13-14$ & $412(46 \%)$ & 9no grado & $290(32.4 \%)$ \\
$15-16$ & $143(16 \%$ & 10 mo grado & $41(4.6 \%)$ \\
$17-18$ & $81(9.1 \%)$ & 11 mo grado & $55(6.1 \%)$ \\
$19-20$ & $26(2.9 \%)$ & 12 mo grado & $70(7.8 \%)$ \\
$21-22$ & $13(1.5 \%)$ & Grado Asociado & $9(1 \%)$ \\
$23-24$ & $9(1 \%)$ & Bachillerato & $19(2.1 \%)$ \\
$25+$ & $79(8.8 \%)$ & Maestría & $13(1.5 \%)$ \\
& & Doctorado & $91(10.2 \%)$ \\
Masculinos & & \\
12 & $193(20 \%)$ & 8vo grado & $413(42.9 \%)$ \\
$13-14$ & $379(39.4 \%)$ & 9no grado & $278(28.9 \%)$ \\
$15-16$ & $211(21.9 \%)$ & 10mo grado & $41(4.3 \%)$ \\
$17-18$ & $100(10.4 \%)$ & 11 mo grado & $70(7.3 \%)$ \\
$19-20$ & $22(2.3 \%)$ & 12mo grado & $86(8.9 \%)$ \\
$21-22$ & $13(1.3 \%)$ & Grado Asociado & $6(0.6 \%)$ \\
$23-24$ & $8(0.8 \%)$ & Bachillerato & $9(0.9 \%)$ \\
$25+$ & $37(38 \%)$ & Maestría & $12(1.2 \%)$ \\
& & Doctorado & $48(5 \%)$ \\
\hline
\end{tabular}

\section{Instrumentos}

Sub-escalas experimentales. Redactamos los ítems de las sub-escalas experimentales partiendo de las concepciones descritas anteriormente. Redactamos 6 actividades para cada sub-escala experimental. Un ejemplo de ítem incluido en la escala de orientación utilitaria es: Cazar animales salvajes. Un ejemplo de ítem de la escala de orientación afectiva es: Cuidar caballos de carrera. Dichas escalas se incluyeron en una versión corta del ICI-PR. 


\section{Procedimientos Generales}

Este inventario experimental se ofreció en línea, libre de costo durante 6 meses, junto a otras pruebas experimentales. Dichas pruebas experimentales impartieron la siguiente instrucción a los participantes: “Los participantes en una prueba experimental pueden llenarla en forma anónima utilizando un seudónimo. Entendemos que al tomar la prueba la persona accede a participar voluntariamente. De cambiar de opinión, puede concluir su participación en cualquier momento. Las pruebas no presentan preguntas que invadan la privacidad de las personas ni tiene ningún efecto negativo. El participante puede abandonar la investigación en cualquier momento. Los resultados informados inicialmente son preliminares, sujetos a cambios menores. Los participantes que interesen que se corrija nuevamente su prueba y se le aplique las normas obtenidas, o que tengan preguntas sobre la prueba experimental, pueden escribirnos a la siguiente dirección electrónica: (Se ofreció la dirección electrónica)."

Al concluir con la recopilación de datos, se realizó un análisis de ítems utilizando el programa Iteman. Realizamos el análisis de ítems de ambas escalas para cada género por separado. Para lograr los dos objetivos de la primera parte, obtuvimos la confiabilidad alfa de las dos escalas de interés en animales y la correlación entre ellas para cada género de participantes. Para evaluar los dos objetivos de la segunda parte correlacionamos ambas escalas de interés en animales y con las escalas de Interés hacia personas e Interés hacia objetos del ICI-PR.

\section{RESULTADOS}

\section{Primera Parte del Estudio}

Los dos objetivos de esta parte eran desarrollar dos sub-escalas de intereses en animales que evaluaran los tipos de orientación (utilitaria y afectiva) de manera confiable y que dichas sub-escalas fueran relativamente independientes una de la otra. Encontramos que ambas sub-escalas resultaron confiables para cada género (Alfa de Cronbach) con coeficientes de .82 y .84 para ambos géneros. El segundo objetivo de que las escalas fueran relativamente independientes una de la otra no se logró, ya que la correlación entre ambas fue de $r=.75$ para los participantes femeninos y .78 para los participantes masculinos, lo que sobrepasa el umbral establecido de $r=.70$ en ambos casos. Esto significa que ambas escalas miden esencialmente el mismo constructo.

\section{Segunda Parte del Estudio}

Los objetivos de esta segunda parte eran validar las dos escalas de interés en animales mediante la correlación de las mismas con las Escalas de orientación primaria del ICI-PR. Se esperaba una correlación significativa entre la escala de orientación utilitaria y la de Orientación hacia objetos. Igualmente, se esperaba una correlación significativa entre la escala de orientación afectiva y la de Orientación hacia personas.

El análisis se hizo para cada género por separado. Las correlaciones de ambas escalas de interés en animales, con ambas escalas de orientación primarias del ICI-PR, fueron estadísticamente significativas y moderadas en las muestras de ambos géneros (véase Tabla 2). Las correlaciones de la escala de orientación utilitaria y orientación afectiva fueron ligeramente más altas con la escala de orientación hacia objetos que la escala de orientación hacia personas. Estos resultados se interpretan en términos de que no hubo evidencia en este estudio de la existencia de dos orientaciones distintas hacia los animales, sino que el interés en animales se relaciona más con la orientación hacia objetos.

\section{Tabla 2}

Correlación entre la escala de interés en animales y escalas de Intereses (ICI-PR).

\begin{tabular}{lcc}
\hline & $\begin{array}{c}\text { Interés hacia } \\
\text { Personas }\end{array}$ & $\begin{array}{c}\text { Interés hacia } \\
\text { objetos }\end{array}$ \\
\hline Femeninos & 0.38 & 0.42 \\
Masculinos & 0.47 & 0.48 \\
Total & 0.43 & 0.44 \\
\hline
\end{tabular}

\section{Ensamblaje de una Escala de Interés en Animales}

Antes de ensamblar la escala realizamos un análisis de ítems para identificar si se debía eliminar reactivos que tuvieran una correlación de poca magnitud con la escala. Los resultados nos llevaron a eliminar 2 ítems con diferencias significativas. Procedimos entonces a ensamblar una sola escala de 10 ítems utilizando igual número de ítems de las escalas utilitaria y afectiva. Los datos básicos para dicha escala se presentan en la Tabla 3. 
Tabla 3

Datos básicos de la escala de interés en animales, versión de 10 items.

\begin{tabular}{cccccc}
\hline & $\mathrm{M}$ & $\mathrm{DE}$ & $\mathrm{Me}$ & $\alpha$ & $\mathrm{EM}$ \\
\hline Femenino & 32.82 & 10.2 & 34 & .90 & 3.25 \\
Masculino & 34.06 & 10.65 & 35 & .91 & 3.23 \\
Total & 33.2 & 10.37 & 34 & .90 & 3.39 \\
\hline
\end{tabular}

Nota: $M=$ media; $\mathrm{DE}=$ Desviación estándar; $\mathrm{Me}=\mathrm{Me}-$ diana; $\alpha=$ Alfa de Cronbach; EM = Error de medición

\section{DISCUSIÓN}

\section{Contribución Teórica}

Nos planteamos la posibilidad de que existan dos tipos de interés en animales: uno con orientación utilitaria y otro con orientación afectiva. Redactamos sub-escalas experimentales para medir ambas orientaciones de intereses y las probamos con una muestra de conveniencia obtenida por internet. Los resultados indicaron que dichas escalas están midiendo esencialmente el mismo constructo, por lo que no podemos establecer orientaciones distintas hacia el interés que muestran las personas hacia los animales. Esto podría incidir sobre cuán complicada es la relación humanoanimal (Gutiérrez et al., 2007). Algo que debería tomarse en consideración es la reducción del consumo de animales (Medawar et al., 2019), el cual podría afectar la manera en la que algunos participantes se relacionan y muestran su interés hacia animales.

Además, correlacionamos ambas sub-escalas experimentales y la escala final resultante con las escalas de interés hacia Personas y Objetos del ICI-PR. Encontramos correlaciones sustanciales en todos los casos para ambos géneros. Estos resultados muestran que la escala de interés en animales probablemente surgió de la orientación primaria hacia los objetos según propone el modelo estructural de intereses presentado por Cirino y Ortiz (2019), sin embargo, sus correlaciones con la orientación primaria de orientación hacia personas deben ser esclarecida mediante investigación.

\section{REFERENCIAS}

Ato, M., López, J. J., \& Benavente, A. (2013). Un sistema de clasificación de los diseños de investigación en psicología. Anales de Psicología, 29(3), 1038-1059. https://doi.org/10.6018/analesps.29.3.178511

\section{Contribución Práctica}

Hay una notable ausencia de inventarios de intereses que incluyan una escala para medir el interés en animales. Esto es necesario porque los intereses están relacionados con la toma de decisiones de carrera, éxito y permanencia académica y ocupacional y a otros indicadores conductuales importante. Este estudio permitió desarrollar un instrumento válido y confiable para evaluar este interés en animales que debe ser útil en el estudio de los intereses y contribuyendo a toma de decisiones recreativas, educativas y de carrera.

\section{Limitaciones}

Jackson y Messick (1958) distinguieron entre estilos de respuestas y disposiciones de respuestas. La deseabilidad social es un estilo de respuestas que ha preocupado mucho a los investigadores y se ha escrito mucho sobre la naturaleza y el control estadístico de este estilo (Bolt et al., (2014), pero controversia ha estado vigente por más de 30 años sin visos de resolverse (Smith \& Ellingson, 2002). Se ha encontrado que los estilos de respuestas no afectan su validez predictiva (Smith \& Ellingson, 2002) y muchos consideran que estos estilos son variables válidas de las preguntas. En este trabajo optamos por aceptar dichas disposiciones como parte de lo que mide un instrumento. Después de todo, los inventarios de intereses sólo incluyen actividades legales y moralmente aceptables y excluyen las que no lo son, por lo que todos los ítems son socialmente aceptables.

\section{CONCLUSIÓN}

Luego de analizar los resultados del presente estudio, decidimos incluir la escala de interés en animales como parte de las escalas básicas de Interés en Objetos en el Inventario Cirino de Intereses.

Financiamiento: La presente investigación no fue financiada por alguna entidad ni patrocinador.

Conflicto de Intereses: No existen conflictos de intereses de parte de los autores de la investigación.

Bolt, D. M., Lu, Y., \& Kim, J.-S. (2014). Measurement and control of response styles using anchoring vignettes: A model-based approach. Psychological Methods, 19(4), 528-541. https://doi.org/10.1037/met0000016 
Cabán Huertas, M., Rosario Nieves, I., \& Álvarez, M. (2014). Desarrollo de la terapia asistida por animales en la psicología. Informes Psicológicos, 14(2), 125-144.

Campbell, D. P., Hyne, S. A., \& Nilsen, D. L. (1992). Manual for the Campbell interest and skill survey: CISS. National Computer Systems.

Carlisle, G. K. (2015). The social skills and attachment to dogs of children with autism spectrum disorder. Journal of Autism and Developmental Disorders, 45(5), 1137-1145. https://doi.org/10.1007/s10803-014-2267-7

Cirino, G. (2013). Origen, desarrollo y naturaleza de los intereses. Palibrio.

Cirino, G., \& Ortiz, L. M. (2019). Hacia un modelo universal de los intereses. Revista Interamericana de Psicología, 53(1), 8-16. https://doi.org/10.30849/rip/ijp.v53i1.128

Fleishman, J. A., Spector, W. D., \& Altman, B. M. (2002). Impact of differential item functioning on age and gender differences in functional disability. The Journals of Gerontology Series B: Psychological Sciences and Social Sciences, 57(5), 275-284. https://doi.org/10.1093/geronb/57.5.s275

Gutiérrez, G., Granados, D. R., \& Piar, N. (2007). Interacciones humano-animal: Características e implicaciones para el bienestar de los humanos. Revista Colombiana de Psicología, 16(1), 163-184.

Hansen, J. I. C. (1995). Interest Assessment. ERIC Digest.

Hansen, J. C. (1990). Interest inventories. Chapter in S. Goldstein \& M. Hersen (eds.). "Handbook of psychological assessment" (pp. 173-194).

Harmon, L. W., Hansen, J. C., Borgen, F. H., \& Hammer, A. L. (1994). Applications and technical guide for the Strong Interest Inventory.
Holland, J. L. (1997). Making vocational choices: A theory of vocational personalities and work environments. Psychological Assessment Resources.

Jackson, D. N., \& Messick, S. (1958). Content and style in personality assessment. Psychological Bulletin, 55(4), 243. https://psycnet.apa.org/doi/10.1037/h0045996

LoBue, V., Bloom Pickard, M., Sherman, K., Axford, C., \& DeLoache, J.S. (2013). Young children's interest in live animals. British Journal of Developmental Psychology, 31(1), 57-69. https://doi.org/10.1111/j.2044-835X.2012.02078.x

Maharaj, N. (2016). Companion animals and vulnerable youth: Promoting engagement between youth and professional service. Journal of Loss and Trauma, 21(4), 335-343. https://doi.org/10.1080/15325024.2015.1084857

Medawar, E., Huhn, S., Villringer, A., \& Witte, A. V. (2019). The effects of plant-based diets on the body and the brain: A systematic review. Translational psychiatry, 9, 226. https://doi.org/10.1038/s41398-019-0552-0

Meléndez-Samó, L. M. (2014). El vínculo humano-animal y sus implicaciones para la psicología en Puerto Rico. Revista Puertorriqueña de Psicología, 25(1), 160-182.

Smith, D. B., \& Ellingson, J. E. (2002). Substance versus style: A new look at social desirability in motivating contexts. Journal of Applied Psychology, 87(2), 211-219. https://doi.org/10.1037/00219010.87.2.211

Younggren, J. N., Boisvert, J. A., \& Boness, C. L. (2016). Examining emotional support animals and role conflicts in professional psychology. Professional Psychology: Research and Practice, 47(4), 255-260. https://dx.doi.org/10.1037\%2Fpro0000083

\section{Obra bajo licencia de Creative Commons Atribución 4.0 Internacional (CC BY 4.0).}

(C) 2020 Autores. 